\title{
A mass conserved splitting method for the nonlinear Schrödinger equation
}

\author{
Dong-Ying Hua ${ }^{1}$, Xiang-Gui $\mathrm{Li}^{1 *}$ and Jiang $\mathrm{Zhu}^{2}$
}

\author{
* Correspondence: xianggui-li@vip. \\ sina.com \\ ${ }^{1}$ School of Applied Science, Beijing \\ Information Science and \\ Technology University, Beijing \\ 100192, P. R. China \\ Full list of author information is \\ available at the end of the article
}

\begin{abstract}
A mass conserved time-splitting difference method is presented for the onedimensional dipolar Bose-Einstein condensates (BECs) described by a nonlocal nonlinear Schrödinger equation with a convolution term. As a result of the singularity in the convolution term, it brings difficulties both in mathematical analysis and in numerical simulations. By properly using the difference scheme to deal with the convolution term, an imaginary time method is given to compute the ground states and then a time-splitting method is obtained for dynamics of dipolar BECs. This time-splitting numerical method is mass conserved everywhere, and it has second-order accuracy and is also unconditionally stable. Numerical results are given to verify the stability and energy conservation when there is no blow up.

Mathematics Subject Classification (2010) 65M06; $35 \mathrm{Q} 41$.
\end{abstract}

Keywords: time-splitting, finite difference, dipolar Bose-Einstein condensates, Schrödinger equation, conservation

\section{Introduction}

Since its first experimental realization in dilute bosonic atomic gases in 1995, the BoseEinstein condensation (BEC) of ultra-cold atomic and molecular gases has attracted considerable interests both theoretically and experimentally. Most of their properties of these trapped quantum gases are governed by the interactions between particles in the condensate [1]. In recent years, there has been an investigation for realizing a new kind of quantum gases with the dipolar interaction, acting between particles having a permanent magnetic or electric dipole moment. A BEC of ${ }^{52} \mathrm{Cr}$ atoms was realized $[2,3]$ at Stuttgart University in 2005, which paves the way towards the experimental as well as the theoretical and the numerical investigation on this novel dipolar quantum gases. The interest on dipolar gases has also been motivated by a broad range of exciting applications [4-6]. In such condensates, there are two kinds of interactions, namely, the short-range repulsive interaction between the particles as well as their long-range partially attractive/partially repulsive dipolar interactions. Therefore, the macroscopic condensate dynamics is determined by a dynamical equilibration of these forces $[7,8]$. The effects of the interparticle interactions on the condensate properties have been widely discussed for the case of short-range interactions. However, since the dipoledipole interactions are long range, anisotropic and partially attractive, the nontrivial task of achieving and controlling dipolar BECs is thus particularly challenging.

(c) 2012 Hua et al; licensee Springer. This is an Open Access article distributed under the terms of the Creative Commons Attribution License (http://creativecommons.org/licenses/by/2.0), which permits unrestricted use, distribution, and reproduction in any medium, provided the original work is properly cited. 
As we know, the properties of a BEC at temperatures $T$ very much smaller than the critical temperature $T_{\mathrm{c}}$ are usually described by the nonlinear Schrödinger (NLS) equation for the macroscopic wave function known as the Gross-Pitaevskii (GP) equation. As far as the dipolar interaction is concerned, a convolution term is introduced [9-11] to modify the classical GP equation, which results in the following differential-integral equation

$$
i \hbar \psi_{t}(\mathbf{x}, t)=\left(-\frac{\hbar}{2 m} \nabla^{2}+V(\mathbf{x})+g|\psi(\mathbf{x}, t)|^{2}+V_{\mathrm{dip}}(\mathbf{x}) *|\psi(\mathbf{x}, t)|^{2}\right) \psi(\mathbf{x}, t),
$$

where $\hbar$ is the Planck constant, $m$ the mass of the atom and $V(\mathbf{x})$ is the external trapping potential, which is generally harmonic, and $g=4 \pi \hbar^{2} a_{s} / m$ is the local interactions between dipoles in the condensate with $a_{\mathrm{s}}$ the $\mathrm{s}$ wave scattering length (positive for repulsive interaction and negative for attractive interaction). $V_{\text {dip }}(\mathbf{x})$ is the long-range isotropic dipolar interaction potential between two dipoles. The wave function $\psi(\mathbf{x}, t)$ is normalized according to

$$
\|\psi\|^{2}=\int_{\mathbb{R}^{3}}|\psi(\mathbf{x}, t)|^{2} \mathrm{~d} \mathbf{x}=N
$$

where $N$ is the number of the atoms in the dipolar BEC. The Equation 1 can be made dimensionless and simplified by adopting a unit system where the units for length, time and energy are given by $a_{0}, 1 / \omega_{0}$, and $\hbar \omega_{0}$, respectively, with $\omega_{0}=\min \left\{\omega_{1}\right.$, $\left.\omega_{2}, \omega_{3}\right\}, a_{0}=\sqrt{\frac{h}{m \omega_{0}}}$. Here, we only consider the one-dimensional (1D) case. Apart from a conceptual clarity, lower dimensional dipolar BECs also offer a clear advantage for numerical computations. And also, in the case of radial symmetry in 2D and spherical symmetry in 3D (by tuning the trap frequencies), the multi-dimensional problem can be reduced to $1 \mathrm{D}$ case.

Now consider the following 1D dimensionless GP equation:

$$
\begin{aligned}
& i \frac{\partial \psi(x, t)}{\partial t}=\left(-\frac{1}{2} \nabla^{2}+V(x)+\beta|\psi(x, t)|^{2}+\lambda V_{\mathrm{dip}}(x) *|\psi(x, t)|^{2}\right) \psi(x, t), \\
& \psi(x, 0)=\psi_{0}(x), \quad x \in \mathbb{R}
\end{aligned}
$$

where $t$ is time, $x$ is displacement, $\beta$ and $\lambda$ are two dimensionless real parameters stand for the strength of the short-range interaction between the particles and the long-range dipolar interaction, respectively. $\psi(x, t): \mathbb{R} \times \mathbb{R}^{+} \rightarrow \mathbb{C}$ is a complexvalued function, $V(x)=x^{2} / 2$ stands for the harmonic potential in $1 \mathrm{D}, V_{\mathrm{dip}}(x)=|x|^{-\alpha}$, $0<\alpha<1$ is the convolution kernel representing the dipolar potential and * is the standard convolution in $\mathbb{R}$, i.e.,

$$
V_{\text {dip }}(x) *|\psi(x, t)|^{2}=\int_{\mathbb{R}} \frac{|\psi(y, t)|^{2}}{|x-y|^{\alpha}} \mathrm{d} y, \quad x, y \in \mathbb{R} .
$$

In order to study the ground states and dynamics of dipolar BECs, an efficient numerical method is necessary. For the study in mathematics, the existence and uniqueness as well as the possible blowup of solutions were studied in [12], and the 
existence of standing waves was proven in $[13,14]$. Due to the dipolar interaction potential of the nonlocal NLS, mathematical and numerical difficulties are introduced. Currently, the Fourier transform $[15,16]$ is generally used for dealing with the convolution in (3). However, there are two limitations in these numerical methods: (i) the Fourier transform of the dipolar interaction potential $V_{\text {dip }}(x)$ and the density function is usually carried out in the whole space $\mathbb{R}$ in the continuous level, while they are carried out on a bounded domain $\Omega$ in the discrete level, so there is a locking phenomena in practical computation as observed in [17]; (ii) the denominator of the dipolar interaction potential equals zero when $x$ equals $y$, thus this singularity may cause some numerical difficulties too. The purpose here is to present a robust method for computing ground states and dynamics of dipolar BECs without these two limitations. The key issue is to deal with the convolution term in the nonlocal NLS equation efficiently.

This paper is organized as follows. In Sect. 2, some analytic results are given for the nonlocal NLS. In Sect. 3, a Crank-Nicolson numerical method is presented for computing ground states of dipolar BECs. In Sect. 4, a time-splitting numerical scheme is proposed for computing the dynamics. Numerical results are reported to verify the efficiency of this numerical method in Sect. 5. Finally, some concluding remarks are drawn in Sect. 6.

\section{Analytical results}

Two important invariants of (3) are the mass (or normalization) of the wave function

$$
N(\psi(x, t)):=\|\psi(x, t)\|^{2}=\int_{\mathbb{R}}|\psi(x, t)|^{2} \mathrm{~d} x \equiv \int_{\mathbb{R}}|\psi(x, 0)|^{2} \mathrm{~d} x=1, \quad t \geq 0,
$$

and the energy of per particle

$$
\begin{aligned}
E(\psi(x, t)): & =\int_{\mathbb{R}}\left\{\frac{1}{2}|\nabla \psi|^{2}+V(x)|\psi|^{2}+\frac{\beta}{2}|\psi|^{4}+\frac{\lambda}{2}\left(V_{\mathrm{dip}}(x) *|\psi|^{2}\right)|\psi|^{2}\right\} \mathrm{d} x \\
& \equiv E(\psi(x, 0)), \quad t \geq 0 .
\end{aligned}
$$

In order to obtain the ground states, we take the ansatz

$$
\psi(x, t)=\mathrm{e}^{-i \mu t} \phi(x), \quad x \in \mathbb{R}, \quad t \geq 0,
$$

where $\mu \in \mathbb{R}$ is the chemical potential and $\varphi:=\varphi(x)$ is a time-independent function. Inserting (8) into (3), we get the time-independent GP equation or the eigenvalue problem

$$
\mu \phi(x)=\left(-\frac{1}{2} \nabla^{2}+V(x)+\beta|\phi(x)|^{2}+\lambda V_{\mathrm{dip}}(x) *|\phi(x)|^{2}\right) \phi(x), \quad x \in \mathbb{R},
$$

under the constraint

$$
\|\phi\|:=\sqrt{\int_{\mathbb{R}}|\phi(x)|^{2} \mathrm{~d} x}=1 .
$$

The ground state of a dipolar BEC is defined as the solution of the following nonconvex minimization problem: 
Find $\varphi_{g} \in S$ and $\mu_{g} \in \mathbb{R}$ such that

$$
E^{g}:=E\left(\phi_{g}\right)=\min _{\phi \in S} E(\phi), \quad \mu^{g}:=\mu\left(\phi_{g}\right),
$$

where the nonconvex set $S$ is defined as

$$
S:=\{\phi(x) \mid\|\phi\|=1, \quad E(\phi)<\infty\} .
$$

And the chemical potential (or eigenvalue of (9)) is defined as

$$
\begin{aligned}
\mu(\phi): & =\int_{\mathbb{R}}\left\{\frac{1}{2}|\nabla \phi|^{2}+V(x)|\phi|^{2}+\beta|\phi|^{4}+\lambda\left(V_{\mathrm{dip}}(x) *|\phi|^{2}\right)|\phi|^{2}\right\} \mathrm{d} x \\
& \equiv E(\phi)+\frac{1}{2} \int_{\mathbb{R}}\left\{\beta|\phi|^{4}+\lambda\left(V_{\mathrm{dip}}(x) *|\phi|^{2}\right)|\phi|^{2}\right\} \mathrm{d} x
\end{aligned}
$$

The total energy in (7) is composed of kinetic, potential, interaction and dipolar energies, respectively, i.e.,

$$
E(\phi)=E_{\mathrm{kin}}(\phi)+E_{\mathrm{pot}}(\phi)+E_{\mathrm{int}}(\phi)+E_{\mathrm{dip}}(\phi),
$$

where

$$
\begin{gathered}
E_{\text {kin }}(\phi)=\frac{1}{2} \int_{\mathbb{R}}|\nabla \phi|^{2} \mathrm{~d} x, \quad E_{\text {pot }}(\phi)=\int_{\mathbb{R}} V(x)|\phi(x)|^{2} \mathrm{~d} x, \\
E_{\text {int }}(\phi)=\frac{\beta}{2} \int_{\mathbb{R}}|\phi(x)|^{4} \mathrm{~d} x, \quad E_{\text {dip }}(\phi)=\frac{\lambda}{2} \int_{\mathbb{R}}\left(V_{\text {dip }}(x) *|\phi(x)|^{2}\right)|\phi|^{2} \mathrm{~d} x,
\end{gathered}
$$

and $\varphi(x)$ defined in (8) is a stationary state of a dipolar BEC.

\section{Numerical method for computing ground states}

In this section, we will propose an implicit numerical method for computing the ground states of a dipolar BEC. In practical computation, we usually truncate the problem (3) and (4) into a bounded computational domain $\Omega$ (chosen as anterval $[-a, a]$ in $1 \mathrm{D}$, with $a$ sufficiently large), with homogeneous Dirichlet boundary condition. There are various numerical methods proposed in literatures for computing the ground states of BEC $[8,9,16-19]$. One of the efficient and popular techniques for the constraint (6) is through the following construction [13]: we choose a time step size $\tau>0$ and set $t_{k}=k \tau$ for $k=0,1, \ldots$. Applying the imaginary time method [18] without considering the constraint (6), and then projecting the solution back to the unit nonconvex set $S$ at the end of each time interval $\left[t_{k}, t_{k+1}\right]$ to satisfy the constraint. Then the function $\varphi(x, t)$ is the solution of the following gradient flow with discrete normalization [19]:

$$
\begin{aligned}
& \frac{\partial \phi(x, t)}{\partial t}=\left\{\frac{1}{2} \nabla^{2}-V(x)-\beta|\phi(x, t)|^{2}-\lambda V_{d i p}(x) *|\phi(x, t)|^{2}\right\} \phi(x, t), \\
& \left.\phi(x, t)\right|_{x \in \partial \Omega}=0, \quad t \geq 0, \\
& \phi\left(x, t_{k+1}\right):=\phi\left(x, t_{k+1}^{+}\right)=\frac{\phi\left(x, t_{k+1}^{-}\right)}{\left\|\phi\left(\cdot, t_{k+1}^{-}\right)\right\|}, x \in \Omega, \quad k \geq 1,
\end{aligned}
$$




$$
\phi(x, 0)=\phi_{0}(x), \quad\left\|\phi_{0}\right\|=1,
$$

where $\phi\left(x, t_{k}^{ \pm}\right)=\lim _{t \rightarrow t_{k} \pm} \phi(x, t)$ and $\varphi_{0}(x)$ is the initial condition.

Suppose $M$ is a positive integer, choose the spatial step $h=2 a / M$, and define $x_{j}=-a$ $+j h, j=0,1, \ldots, M$. Let $F_{j}^{k}$ be the approximation of $V_{\text {dip }}\left(x_{j}\right) *\left|\varphi\left(x_{j}, t_{k}\right)\right|^{2}$, and $\phi_{j}^{k}$ be the approximations of $\left(x_{j}, t_{k}\right)$, which are the exact solution of (15)-(18) at the mesh grid $\left(x_{j}, t_{k}\right)$.

Choose $\phi_{j}^{0}=\phi_{0}\left(x_{j}\right), j=0,1, \ldots, M$. From time $t_{k}$ to $t_{k+1}$, a cental difference discretization for (15)-(18) is

$$
\frac{\phi_{j}^{k+1}-\phi_{j}^{k}}{\tau}=\frac{1}{2 h^{2}} \delta_{x}^{2}\left(\phi_{j}^{k+1 / 2}\right)-V\left(x_{j}\right) \phi_{j}^{k+1 / 2}-\beta\left(\left|\phi_{j}^{k}\right|^{2}\right) \phi_{j}^{k+1 / 2}-\lambda F_{j}^{k} \phi_{j}^{k+1 / 2}, \quad j=0,1, \ldots, M,
$$

where

$$
\begin{aligned}
\phi_{j}^{k+1 / 2}= & \left(\phi_{j}^{k+1}+\phi_{j}^{k}\right) / 2, \quad \delta_{x}^{2}\left(\phi_{j}^{k}\right)=\phi_{j+1}^{k}-2 \phi_{j}^{k}+\phi_{j-1}^{k}, \\
F_{j}^{k} & =\sum_{r=0}^{M-1}\left(\left.\frac{1}{1-\alpha}\left|\phi\left(x_{r}, t_{k}\right)\right|^{2}||(r+1-j) h\right|^{1-\alpha}-|(r-j) h|^{1-\alpha} \mid+\frac{\left|\phi\left(x_{r+1}, t_{k}\right)\right|^{2}-\left|\phi\left(x_{r-1}, t_{k}\right)\right|^{2}}{2 h} .\right. \\
& \left.\int_{0}^{h} \frac{s}{|(r-j) h+s|^{\alpha}} \mathrm{d} s+\frac{\left|\phi\left(x_{r+1}, t_{k}\right)\right|^{2}-2\left|\phi\left(x_{r}, t_{k}\right)\right|^{2}+\left|\phi\left(x_{r-1}, t_{k}\right)\right|^{2}}{h^{2}} \cdot \int_{0}^{h} \frac{s^{2}}{2|(r-j) h+s|^{\mid}} \mathrm{d} s\right),
\end{aligned}
$$

and the details of $F_{j}^{k}$ are given in the Appendix. Notice that in the above scheme, we replace $\beta\left(\left|\phi_{j}^{k+1 / 2}\right|^{2}\right) \phi_{j}^{k+1 / 2}, F_{j}^{k+1 / 2}$ with $\beta\left(\left|\phi_{j}^{k}\right|^{2}\right) \phi_{j}^{k+1 / 2}$ and $F_{j}^{k}$, respectively, to linearize the nonlinear terms. It is easy to see that the local truncation error of (19) is $O\left(h^{2}+\tau\right.$ ${ }^{2}$ ) because $0<\alpha<1$. Using the classical finite difference method theory, we claim our numerical scheme is the second-order algorithm which will be justified by the numerical examples in Sect. 5.

\section{Time-splitting numerical method for dynamics}

We will propose a time-splitting finite difference (TSFD) method for computing the dynamics of a dipolar BEC based on the nonlocal NLS equation (3). The advantage of this method is to deal with the discretization of nonlinear terms in the NLS equation by solving an ordinary differential equation (ODE) exactly. By virtue of this way, the computational cost and complexity can be reduced. As in Sect. 3, the whole space problem is truncated into a bounded computational domain $\Omega=[-a, a]$ with homogeneous Dirichlet boundary condition.

From $t=t_{k}$ to $t=t_{k+1}$, the GP equation (3)-(4) is solved by three steps. First, we solve

$$
\begin{aligned}
& i \frac{\partial \psi(x, t)}{\partial t}=-\frac{1}{2} \nabla^{2} \psi(x, t), \quad x \in \Omega, \\
& \psi(-a, t)=0, \quad \psi(a, t)=0,
\end{aligned}
$$

from $t_{k}$ to $t_{k+1 / 2}$, followed by solving the nonlinear ODE

$$
i \frac{\partial \psi(x, t)}{\partial t}=\left(V(x)+\beta|\psi(x, t)|^{2}+\lambda V_{\mathrm{dip}}(x) e s *|\psi(x, t)|^{2}\right) \psi(x, t),
$$




$$
\psi(-a, t)=0, \psi(a, t)=0, t_{k} \leq t \leq t_{k+1}
$$

for one time step. Again, we slove (21) from $t_{k+1 / 2}$ to $t_{k+1}$.

Equation 21 can be discretized in space by Crank-Nicolson scheme, and Equation 23 can be solved exactly. In fact, for $t \in\left[t_{k}, t_{k+1}\right]$, multiplying (23) by the conjugation of $\psi(x, t)$, i.e., $\overline{\psi(x, t)}$, we get

$$
i \frac{\partial \psi(x, t)}{\partial t} \overline{\psi(x, t)}=\left(V(x)+\beta|\psi(x, t)|^{2}+\lambda V_{\mathrm{dip}}(x) *|\psi(x, t)|^{2}\right) \psi(x, t) \overline{\psi(x, t)},
$$

and we also have

$$
-i \frac{\partial \overline{\psi(x, t)}}{\partial t} \psi(x, t)=\left(V(x)+\beta|\psi(x, t)|^{2}+\lambda V_{\text {dip }}(x) *|\psi(x, t)|^{2}\right) \overline{\psi(x, t)} \psi(x, t),
$$

Therefore, subtracting (26) from (25), one obtains

$$
i \frac{\mathrm{d}}{\mathrm{d} t}|\psi(x, t)|^{2}=0 .
$$

which implies

$$
|\psi(x, t)|^{2}=\left|\psi\left(x, t_{k}\right)\right|^{2}, \quad t_{k} \leq t \leq t_{k+1} .
$$

Substituting (27) into (23), we get a linear ODE

$$
i \frac{\partial \psi(x, t)}{\partial t}=\left(V(x)+\beta\left|\psi\left(x, t_{k}\right)\right|^{2}+\lambda V_{\mathrm{dip}}(x) *\left|\psi\left(x, t_{k}\right)\right|^{2}\right) \psi(x, t)
$$

which can be solved exactly. Integrating (28) from $t_{k}$ to $t$, one gets

$$
\psi(x, t)=\exp \left\{-i\left[V(x)+\beta\left|\psi\left(x, t_{k}\right)\right|^{2}+\lambda V_{d i p}(x) *\left|\psi\left(x, t_{k}\right)\right|^{2}\right]\left(t-t_{k}\right)\right\} \psi\left(x, t_{k}\right), \quad t_{k} \leq t \leq t_{k+1} .
$$

Let $\psi_{j}^{k}$ be the approximation of $\psi\left(x_{j}, t_{k}\right)$. Then a second-order TSFD method for solving (3)-(4) via the standard Strang splitting [20-22] is as follows:

$$
\left\{\begin{array}{l}
\psi_{j-1}^{(1)}+\left(4 i / \lambda_{0}-2\right) \psi_{j}^{(1)}+\psi_{j+1}^{(1)}=-\psi_{j-1}^{k}+\left(4 i / \lambda_{0}+2\right) \psi_{j}^{k}-\psi_{j+1}^{k}, \\
\psi_{j}^{(2)}=\exp \left\{-i \tau\left[V\left(x_{j}\right)+\beta\left|\psi_{j}^{(1)}\right|^{2}+\lambda F_{j}^{(1)}\right]\right\} \psi_{j}^{(1)}, j=1,2, \ldots, M-1, \\
\psi_{j-1}^{k+1}+\left(4 i / \lambda_{0}-2\right) \psi_{j}^{k+1}+\psi_{j+1}^{k+1}=-\psi_{j-1}^{(2)}+\left(4 i / \lambda_{0}+2\right) \psi_{j}^{(2)}-\psi_{j+1}^{(2)}
\end{array}\right.
$$

where $\lambda_{0}=\tau / 2 h^{2}, \psi_{M}=0$ and $F_{j}^{(1)}=V_{\text {dip }}\left(x_{j}\right) *\left|\psi_{j}^{(1)}\right|^{2}$.

The above method is implicit, unconditionally stable. In fact, for the stability or conservation, we have

Theorem 1. The scheme (30) is normalization conservation, i.e.,

$$
\left\|\psi^{k+1}\right\|_{l^{2}}^{2}:=h \sum_{j=0}^{M}\left|\psi_{j}^{k+1}\right|^{2} \equiv h \sum_{j=0}^{M}\left|\psi_{j}^{0}\right|^{2}=\left\|\psi^{0}\right\|_{l^{2}}^{2}, \quad k \geq 0 .
$$

Proof. According to the first equation of (30), if we denote $A_{j}=\psi_{j}^{(1)}+\psi_{j}^{k}$, we have

$$
\frac{4 i}{\lambda_{0}}\left(\psi_{j}^{(1)}-\psi_{j}^{k}\right)=-\left(A_{j-1}+A_{j+1}\right)+2 A_{j} .
$$


Multiplying (32) by the conjugation of $A_{j}$, one gets

$$
\frac{4 i}{\lambda_{0}}\left(\left|\psi_{j}^{(1)}\right|^{2}+\psi_{j}^{(1)} \psi_{j}^{k *}-\psi_{j}^{(1) *} \psi_{j}^{k}-\left|\psi_{j}^{k}\right|^{2}\right)=-\left(A_{j-1}+A_{j+1}\right) A_{j}^{*}+2\left|A_{j}\right|^{2} .
$$

Therefore

$$
-\frac{4 i}{\lambda_{0}}\left(\left|\psi_{j}^{(1)}\right|^{2}+\psi_{j}^{(1) *} \psi_{j}^{k}-\psi_{j}^{(1)} \psi_{j}^{k *}-\left|\psi_{j}^{k}\right|^{2}\right)=-\left(A_{j-1}^{*}+A_{j+1}^{*}\right) A_{j}+2\left|A_{j}\right|^{2} .
$$

Combine the above two equations, we have

$$
\frac{8 i}{\lambda_{0}}\left(\left|\psi_{j}^{(1)}\right|^{2}-\left|\psi_{j}^{k}\right|^{2}\right)=-\left(A_{j-1}+A_{j+1}\right) A_{j}^{*}+\left(A_{j-1}^{*}+A_{j+1}^{*}\right) A_{j}
$$

Then let $j$ be $1,2, \ldots, M-1$, respectively, in (34) and add them together, it is easy to see that, the right-hand side vanishes due to the homogeneous Dirichlet boundary condition $\psi_{0}=\psi_{M}=0$.

Therefore, we have the following equation

$$
\sum_{j=1}^{M-1}\left|\psi_{j}^{(1)}\right|^{2}=\sum_{j=1}^{M-1}\left|\psi_{j}^{k}\right|^{2} .
$$

Similarly, the following equation is obtained from the third equation of (30),

$$
\sum_{j=1}^{M-1}\left|\psi_{j}^{k+1}\right|^{2}=\sum_{j=1}^{M-1}\left|\psi_{j}^{(2)}\right|^{2}
$$

Based on Equation (29) and the second equation of (30), we have

$$
\sum_{j=1}^{M-1}\left|\psi_{j}^{(2)}\right|^{2}=\sum_{j=1}^{M-1}\left|\exp \left\{-i \tau\left[V\left(x_{j}\right)+\left|\psi_{j}^{(1)}\right|^{2}+\lambda F_{j}^{(1)}\right]\right\} \psi_{j}^{(1)}\right|^{2}=\sum_{j=1}^{M-1}\left|\psi_{j}^{(1)}\right|^{2} .
$$

Combining (35), (36) with (37), we have

$$
\sum_{j=1}^{M-1}\left|\psi_{j}^{k+1}\right|^{2}=\sum_{j=1}^{M-1}\left|\psi_{j}^{k}\right|^{2}
$$

Owing to the boundary condition, we have $\left\|\psi^{k+1}\right\|_{l^{2}}^{2}=\left\|\psi^{k}\right\|_{l^{2}}^{2}$, which implies the $l^{2}$ stable property, i.e., $\left\|\psi^{k+1}\right\|_{l^{2}} \equiv\left\|\psi^{0}\right\|_{l^{2}}, k \geq 0$.

\section{Numerical results}

In this section, we first evaluate numerically the convolution term and then report ground states and dynamics of dipolar BECs using our numerical method.

\subsection{Error for the convolution term}

To test the accuracy of this numerical method, correct computation of the convolution term is important. We take $\varphi(x)=x^{3 / 2}$ for example, as the convolution term $V_{\text {dip }}$ " $\mid \varphi$ $\left.(x)\right|^{2}$ can be computed exactly in this case. Based on our numerical method, i.e., (20), the numerical result shows at least the second-order accuracy. And in fact, it is almost 
the third-order accuracy. We choose $\alpha=0.5$ and solve this problem on $[0,10]$. Table 1 shows the maximum errors with different sizes $h$.

From Table 1, one can see that our new method has a higher order accuracy between the second-order and the third-order in space for evaluating the convolution term.

\subsection{Ground states of dipolar BECs}

Using numerical scheme (15)-(18), the ground states of a dipolar BEC with different parameters are given. The initial condition is $\phi_{0}(x)=\frac{1}{\pi^{1 / 4}} e^{-x^{2} / 2}$. We solve this problem on $[-16,16]$ with $h=1 / 8$ and $\tau=0.001 . \varphi_{g}:=\varphi^{k+1}$ is reached numerically when

$$
\left\|\phi^{k+1}-\phi^{k}\right\|_{\infty}:=\max _{0 \leq j \leq M}\left|\phi_{j}^{k+1}-\phi_{j}^{k}\right| \leq \varepsilon:=10^{-6}
$$

in (15). Figure 1 shows the plot of the ground state $\varphi_{g}(x)$, of a dipolar BEC with $\lambda=$ -0.2 and $\beta=0.5$. Table 2 shows the energy $E^{g}$, chemical potential $\mu^{g}$, kinetic energy $E_{\text {kin }}^{g}:=E_{\text {kin }}\left(\phi_{g}\right)$, potential energy $E_{\text {pot }}^{g}:=E_{\text {pot }}\left(\phi_{g}\right)$, interaction energy $E_{\text {int }}^{g}:=E_{\text {int }}\left(\phi_{g}\right)$, dipolar energy $E_{\mathrm{dip}}^{g}:=E_{\mathrm{dip}}\left(\phi_{g}\right)$, and the maximum value of the wave function $\varphi_{g}(0)$ with $\alpha=0.5$ and potential $V(x)=x^{2} / 2$ for different $\lambda$ and $\beta$ with fixed $\lambda / \beta=-0.4$; Table 3 gives computational results with $\beta=0.5$ for different values of $-0.5 \leq \lambda / \beta \leq 1$. In addition, since the GP equation has no analytical solution, we take the numerical solution in a finer mesh with $M=2048$ and $\tau=0.001$ as the "exact" solution, and the maximum error are given in Table 4.

From Tables 2, 3, 4, and Figure 1, we make the following conclusions: when $\lambda$ increases or $\beta$ decreases with fixed $\lambda / \beta=-0.4$, the maximum value of the wave function $\varphi_{g}(0)$, kinetic energy $E_{\text {kin }}^{g}$, dipolar energy $E_{\text {dip }}^{g}$, energy $E^{g}$ as well as the chemical potential $\mu^{g}$ increase; the potential energy $E_{\mathrm{pot}}^{g}$, interaction energy $E_{\mathrm{int}}^{g}$, and the radius mean square $x_{\mathrm{rms}}^{g}$, defined by $x_{\mathrm{rms}}=\sqrt{\int_{\mathbb{R}} x^{2}\left|\phi_{g}(x)\right|^{2} \mathrm{~d} x}$ decrease (cf. Table 2). For fixed $\beta$, when the ratio $\lambda / \beta$ increases from -0.5 to $1, E_{\mathrm{pot}}^{g}, E_{\mathrm{dip}}^{g}, E^{g} \mu^{g}$, and $x_{\mathrm{rms}}$ of the ground states increase; the maximum value of the wave function $\varphi_{g}(0), E_{\mathrm{kin}}^{g}$, and $E_{\mathrm{int}}^{g}$ decrease (cf. Table 3). This numerical method is second-order accurate and can compute the ground states efficiently (cf. Figure 1; Table 4).

\subsection{Dynamics of dipolar BECs}

By applying our numerical method (30), the dynamics of a dipolar BEC is considered. We apply the bounded computational domain $[-8,8], M=128$, i.e., $h=1 / 8$, time step $\tau=0.01$. The initial data is chosen the same as in the case of computing the ground state of a dipolar BEC.

Table 1 Comparison for evaluating the convolution term under varying spatial sizes $\boldsymbol{h}$

\begin{tabular}{|c|c|c|c|c|c|}
\hline Mesh & $h=1 / 10$ & $h=1 / 20$ & $h=1 / 40$ & $h=1 / 80$ & $h=1 / 160$ \\
\hline Error & $2.105044 \mathrm{e}-3$ & $2.696346 \mathrm{e}-4$ & $3.417339 e-5$ & $4.306438 \mathrm{e}-6$ & $5.409597 \mathrm{e}-7$ \\
\hline
\end{tabular}




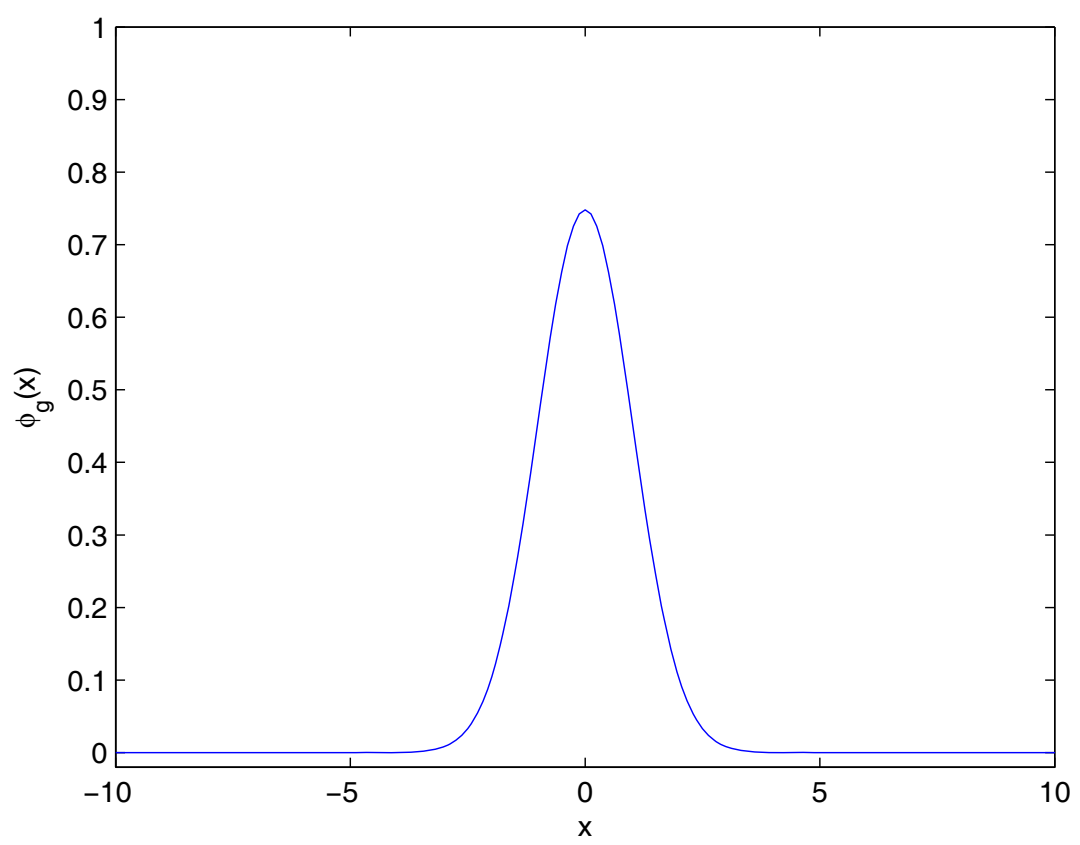

Figure 1 The ground state of a dipolar BEC with $\lambda=-0.2$ and $\beta=0.5$.

Figure 2 depicts the evolution of kinetic energy $E_{\text {kin }}(t):=E_{\text {kin }}(\psi(\cdot, t))$, potential energy $E_{\mathrm{pot}}(t):=E_{\mathrm{pot}}(\psi(\cdot, t))$, interaction energy $E_{\text {int }}(t):=E_{\text {int }}(\psi(\cdot, t))$, dipolar energy $E_{\text {dip }}(t):=E_{\text {dip }}$ $(\psi(, t))$, the energy $E(t):=E(\psi(\cdot, t))$ as well as the chemical potential $\mu(t):=\mu(\psi(\cdot, t))$, condensate width $\sigma_{x}:=\sigma_{x}(\psi(\cdot, t))=\int_{\mathbb{R}} x^{2}|\psi(x, t)|^{2} \mathrm{~d} x$, and $l_{2}$ norm $\|\psi(\cdot, t)\|_{l^{2}}$ for the case of $\beta=5.0$ and $\lambda=-0.8$. In addition, Figure 3 shows the results for the case of $\beta=25$ and $\lambda=0.8$. Figure 4 shows the results for the case of $\beta=-25$ and $\lambda=80$.

From Figures 2, 3, and 4, we can obtain the conclusion that global existence of the solution is observed in the first two cases (cf. Figures 2,3) and finite time blow-up is observed in the last case (cf. Figure 4). The total energy is numerically conserved in our computation when there is no blow-up (cf. Figures 2,3) and the discrete $l^{2}$ norm $\|\psi\|$ is numerically conserved very well whether there is blow-up or not (cf. Figures 2, 3, 4).

Table 2 The ground states of a dipolar BEC for different $\lambda$ and $\beta$ with fixed values of $\lambda / \beta=-0.4$.

\begin{tabular}{lllllllll}
\hline $\boldsymbol{\lambda} \boldsymbol{\beta} \boldsymbol{\beta}$ & $\boldsymbol{\varphi}_{\boldsymbol{g}}(\mathbf{0})$ & $E_{\mathrm{kim}}^{g}$ & $E_{\mathrm{pot}}^{g}$ & $E_{\mathrm{int}}^{g}$ & $E_{\mathrm{dip}}^{g}$ & $\boldsymbol{E}^{g}$ & $\boldsymbol{\mu}^{g}$ & $x_{\mathrm{rms}}^{g}$ \\
\hline$\lambda=-0.2, \beta=0.5$ & 0.747990 & 0.247231 & 0.252333 & 0.099135 & -0.171511 & 0.427188 & 0.354812 & 0.710398 \\
$\lambda=-0.1, \beta=0.25$ & 0.749946 & 0.248765 & 0.250757 & 0.049763 & -0.085918 & 0.463367 & 0.427212 & 0.708176 \\
$\lambda=-0.02, \beta=0.05$ & 0.751125 & 0.249513 & 0.250000 & 0.009974 & -0.017201 & 0.492285 & 0.485058 & 0.707106 \\
$\lambda=0.02, \beta=-0.05$ & 0.751581 & 0.249878 & 0.249634 & -0.009983 & 0.017208 & 0.506737 & 0.513963 & 0.706589 \\
$\lambda=0.1, \beta=-0.25$ & 0.753250 & 0.251210 & 0.248312 & -0.050082 & 0.086181 & 0.535622 & 0.571721 & 0.704716 \\
$\lambda=0.2, \beta=-0.5$ & 0.755449 & 0.253038 & 0.246533 & -0.100613 & 0.172734 & 0.571693 & 0.643814 & 0.702187 \\
\hline
\end{tabular}


Table 3 The ground states of a dipolar BEC with different values of $\lambda / \beta$ for $\beta=0.5$

\begin{tabular}{lllllllll}
\hline $\boldsymbol{\lambda} / \boldsymbol{\beta}$ & $\boldsymbol{\varphi}_{\boldsymbol{g}}(\mathbf{0})$ & $E_{\mathrm{kim}}^{g}$ & $E_{\mathrm{pot}}^{g}$ & $E_{\text {int }}^{g}$ & $E_{\mathrm{dip}}^{g}$ & $\boldsymbol{E}^{g}$ & $\boldsymbol{\mu}^{\boldsymbol{g}}$ & $\boldsymbol{x}_{\mathrm{rms}}^{g}$ \\
\hline-0.5 & 0.751923 & 0.251344 & 0.248178 & 0.100052 & -0.215360 & 0.384214 & 0.268906 & 0.704526 \\
-0.2 & 0.738615 & 0.237322 & 0.262981 & 0.096925 & -0.084809 & 0.512420 & 0.524537 & 0.725233 \\
0 & 0.729516 & 0.228042 & 0.273869 & 0.094808 & 0.000000 & 0.596719 & 0.691527 & 0.740093 \\
0.2 & 0.720684 & 0.219377 & 0.284956 & 0.092781 & 0.083002 & 0.680116 & 0.855899 & 0.754926 \\
0.5 & 0.707932 & 0.207437 & 0.301936 & 0.089903 & 0.204307 & 0.803583 & 1.097793 & 0.777092 \\
1 & 0.688010 & 0.190073 & 0.330997 & 0.085519 & 0.398668 & 1.005257 & 1.489444 & 0.813631 \\
\hline
\end{tabular}

Table 4 The maximum error of the ground state with different mesh

\begin{tabular}{llll}
\hline$M$ & $M=256$ & $M=512$ & $M=1024$ \\
Max-error & $4.36 \mathrm{e}-4$ & $1.04 \mathrm{e}-4$ & $2.10 \mathrm{e}-5$ \\
\hline
\end{tabular}
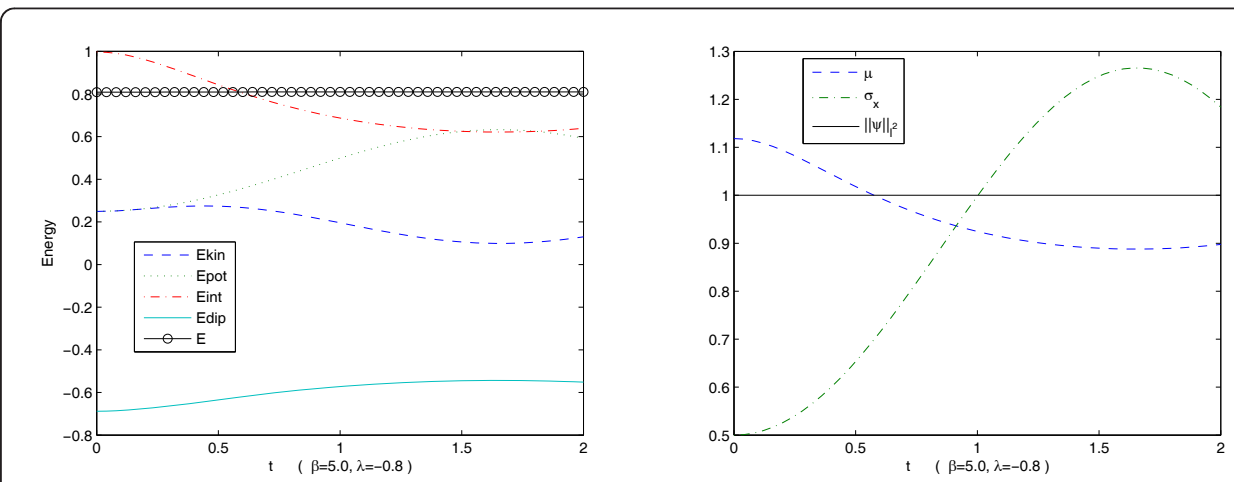

Figure 2 Time evolution at different times for a dipolar BEC with $\beta=5.0$ and $\lambda=-0.8$.

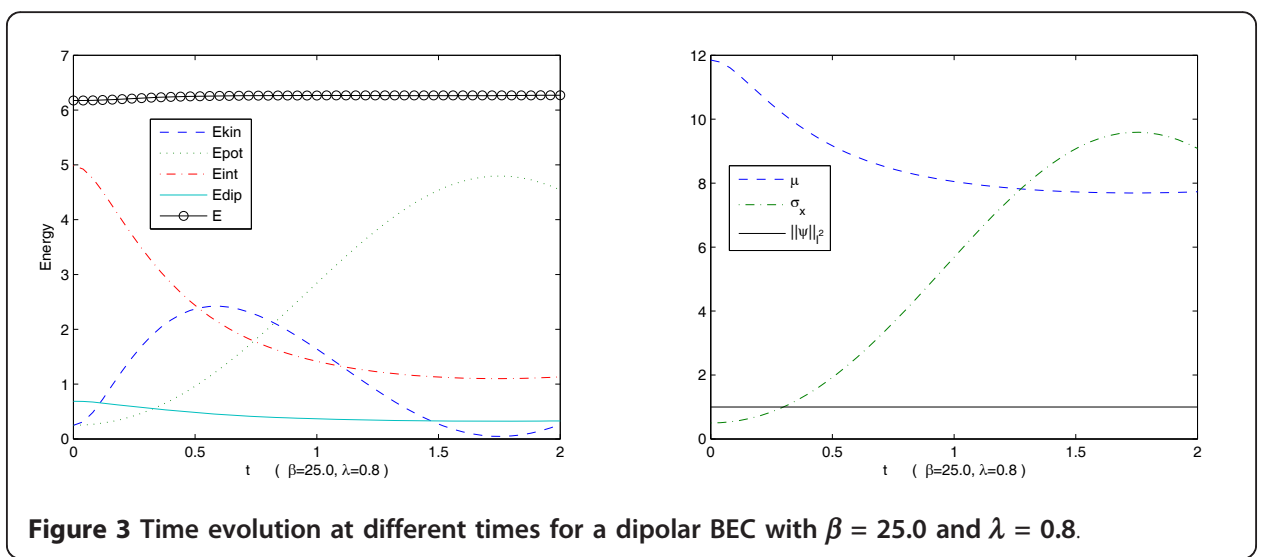

\section{Conclusions}

Efficient numerical methods are presented for computing ground states and dynamics of dipolar Bose-Einstein condensates based on the one-dimensional Grosss-Pitaevskii equation with a nonlocal dipolar interaction potential. By applying the difference 

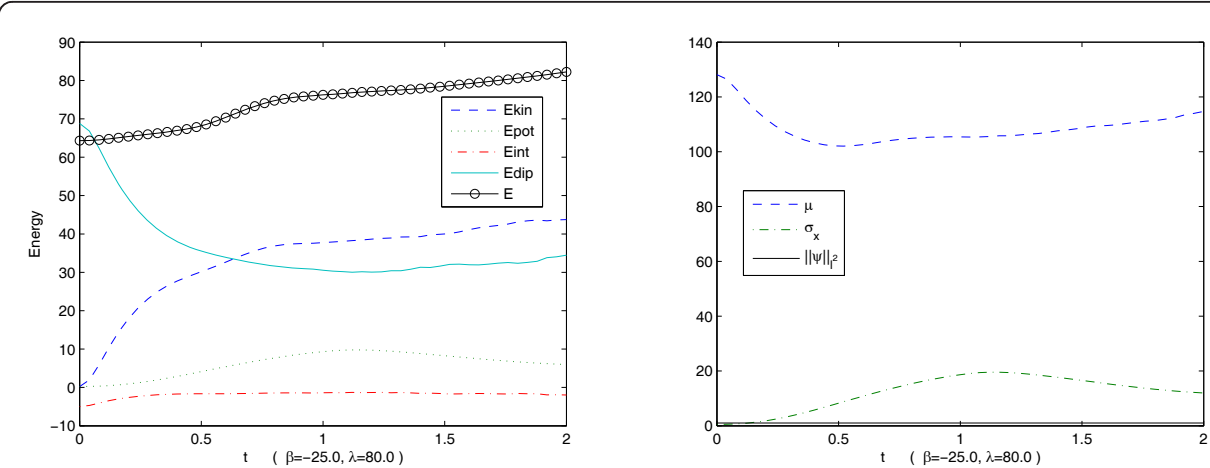

Figure 4 Time evolution for a dipolar BEC with $\beta=-25.0$ and $\lambda=80.0$

method, the discretization of the dipolar interaction potential term is given. The imaginary method and time-splitting difference method are given for computing the ground states and dynamics of a dipolar BEC, respectively. Our numerical method is mass conserved and second-order accurate. In addition, the proof of the discrete energy conservation is an open problem. We only find the energy conservation from a numerical point of view. Numerical results are given to demonstrate the efficiency of our numerical method.

\section{Appendix}

The discretization of the convolution term

Here, we need to handle the convolution term

$$
V_{\text {dip }}(x) *|\psi(x, t)|^{2}=|x|^{-\alpha} *|\psi|^{2}=\int_{R} \frac{1}{|x-y|^{\alpha}}|\psi(y, t)|^{2} \mathrm{~d} y
$$

First, consider $\left.|x|^{-\alpha} *|\psi|^{2}\right|_{\left(x_{j}, t_{k}\right)}$, i.e., $\int_{x_{0}}^{x_{M}} \frac{\mid \psi\left(y_{1}, t_{k}\right)^{2}}{\left|x_{j}-\gamma\right|^{\alpha}} \mathrm{d} y$ where $\left[x_{0}, x_{M}\right]$ is the discretizised integration area. It is easy to see that $x_{j}$ is a singular point and we need to deal with it piecewisely. We denote $I=\int_{x_{0}}^{x_{M}} \frac{\mid \psi\left(\gamma, t_{k}\right)^{2}}{\left|x_{j}-\gamma\right|^{\alpha}} \mathrm{d} y$, let the convolution term be two parts, we have

$$
\int_{x_{0}}^{x_{M}} \frac{\left|\phi\left(y, t_{k}\right)\right|^{2}}{\left|x_{j}-y\right|^{\alpha}} \mathrm{d} y=I_{1}+I_{2}
$$

where

$$
I_{1}=\int_{x_{0}}^{x_{j}} \frac{\left|\phi\left(y, t_{k}\right)\right|^{2}}{\left|x_{j}-\gamma\right|^{\alpha}} \mathrm{d} y, \quad I_{2}=\int_{x_{j}}^{x_{M}} \frac{\left|\phi\left(y, t_{k}\right)\right|^{2}}{\left|x_{j}-\gamma\right|^{\alpha}} \mathrm{d} y .
$$

For $I_{1}$ in (A.2), let $y=x_{r}+s$, we have

$$
I_{1}=\sum_{r=0}^{j-1} \int_{x_{r}}^{x_{r+1}} \frac{\left|\phi\left(y, t_{k}\right)\right|^{2}}{\left(x_{j}-\gamma\right)^{\alpha}} \mathrm{d} y=\sum_{r=0}^{j-1} \int_{0}^{h} \frac{\left|\phi\left(x_{r}+s, t_{k}\right)\right|^{2}}{\left(x_{j}-x_{r}-s\right)^{\alpha}} \mathrm{d} s .
$$


Then, substituting $\left|\varphi\left(x_{r}+s, t_{k}\right)\right|^{2}$ by the Taylor expansion at $\left(x_{r}, t_{k}\right)$, we obtain

$$
\begin{aligned}
I_{1}= & \sum_{r=0}^{j-1} \int_{0}^{h} \frac{1}{\left(x_{j}-x_{r}-s\right)^{\alpha}}\left[\left|\phi\left(x_{r}, t_{k}\right)\right|^{2}+\left.\frac{\partial\left(\left|\phi\left(s, t_{k}\right)\right|^{2}\right)}{\partial s}\right|_{s=x_{r}} s+\left.\frac{\partial^{2}\left(\left|\phi\left(s, t_{k}\right)\right|^{2}\right)}{\partial s^{2}}\right|_{s=x_{r}} \frac{1}{2} s^{2}+O\left(s^{3}\right)\right] \mathrm{d} s \\
= & \sum_{r=0}^{j-1}\left[\left.\frac{-1}{1-\alpha}\left|\phi\left(x_{r}, t_{k}\right)\right|^{2}\left(x_{j}-x_{r}-s\right)^{1-\alpha}\right|_{0} ^{h}+\left.\frac{\partial\left(\left|\phi\left(s, t_{k}\right)\right|^{2}\right)}{\partial s}\right|_{s=x} \int_{r_{0}}^{h} \frac{s}{\left(x_{j}-x_{r}-s\right)^{\alpha}} \mathrm{d} s\right. \\
& \left.+\left.\frac{\partial^{2}\left(\left|\phi\left(s, t_{k}\right)\right|^{2}\right)}{\partial s^{2}}\right|_{s=x_{r}} \int_{0}^{h} \frac{s^{2}}{2\left(x_{j}-x_{r}-s\right)^{\alpha}} \mathrm{d} s+O\left(h^{4-\alpha}\right)\right] \\
= & \sum_{r=0}^{j-1}\left(\frac{1}{1-\alpha}\left|\phi\left(x_{r}, t_{k}\right)\right|^{2}\left\{[(j-r) h]^{1-\alpha}-[(j-r-1) h]^{1-\alpha}\right\}\right. \\
& +\left[\frac{\left|\phi\left(x_{r+1}, t_{k}\right)\right|^{2}-\left|\phi\left(x_{r-1}, t_{k}\right)\right|^{2}}{2 h}+O\left(h^{2}\right)\right] \int_{0}^{h} \frac{s}{\left(x_{j}-x_{r}-s\right)^{\alpha}} \mathrm{d} s \\
& \left.+\left[\frac{\left|\phi\left(x_{r+1}, t_{k}\right)\right|^{2}-2\left|\phi\left(x_{r}, t_{k}\right)\right|^{2}+\left|\phi\left(x_{r-1}, t_{k}\right)\right|^{2}}{h^{2}}+O\left(h^{2}\right)\right] \int_{0}^{h} \frac{s^{2}}{2\left(x_{j}-x_{r}-s\right)^{\alpha}} \mathrm{d} s+O\left(h^{4-\alpha}\right)\right) \\
= & \sum_{r=0}^{j-1}\left(\frac{1}{1-\alpha}\left|\phi\left(x_{r}, t_{k}\right)\right|^{2}\left\{[(j-r) h]^{1-\alpha}-[(j-r-1) h]^{1-\alpha}\right\}\right. \\
& +\left[\frac{\left|\phi\left(x_{r+1}, t_{k}\right)\right|^{2}-\left|\phi\left(x_{r-1}, t_{k}\right)\right|^{2}}{2 h}\right] \int_{0}^{h} \frac{s}{[(j-r) h-s]^{\alpha}} \mathrm{d} s \\
& \left.+\left[\frac{\left|\phi\left(x_{r+1}, t_{k}\right)\right|^{2}-2\left|\phi\left(x_{r}, t_{k}\right)\right|^{2}+\left|\phi\left(x_{r-1}, t_{k}\right)\right|^{2}}{h^{2}}\right] \int_{0}^{h} \frac{s^{2}}{2[(j-r) h-s]^{\alpha}} \mathrm{d} s\right)+O\left(h^{3-\alpha}\right) .
\end{aligned}
$$

Similarly, for $I_{2}$ in (A.2), we get

$$
\begin{aligned}
I_{2}=\sum_{r=j}^{M-1} & \left(\frac{1}{1-\alpha}\left|\phi\left(x_{r}, t_{k}\right)\right|^{2}\left\{[(r+1-j) h]^{1-\alpha}-[(r-j) h]^{1-\alpha}\right\}\right. \\
+ & {\left[\frac{\left|\phi\left(x_{r+1}, t_{k}\right)\right|^{2}-\left|\phi\left(x_{r-1}, t_{k}\right)\right|^{2}}{2 h}\right] \int_{0}^{h} \frac{s}{[(r-j) h+s]^{\alpha}} \mathrm{d} s } \\
+ & {\left.\left[\frac{\left|\phi\left(x_{r+1}, t_{k}\right)\right|^{2}-2\left|\phi\left(x_{r}, t_{k}\right)\right|^{2}+\left|\phi\left(x_{r-1}, t_{k}\right)\right|^{2}}{h^{2}}\right] \int_{0}^{h} \frac{s^{2}}{2[(r-j) h+s]^{\alpha}} \mathrm{d} s\right)+O\left(h^{3-\alpha}\right) . }
\end{aligned}
$$

Combining (A.1), (A.3) and (A.4), we obtain the discretization of convolution term

$$
\begin{aligned}
F_{j}^{k}= & \sum_{r=0}^{M-1}\left(\left.\frac{1}{1-\alpha}\left|\phi\left(x_{r}, t_{k}\right)\right|^{2}||(r+1-j) h\right|^{1-\alpha}-|(r-j) h|^{1-\alpha} \mid\right. \\
& +\left[\frac{\left|\phi\left(x_{r+1}, t_{k}\right)\right|^{2}-\left|\phi\left(x_{r-1}, t_{k}\right)\right|^{2}}{2 h}\right] \int_{0}^{h} \frac{s}{|(r-j) h+s|^{\alpha}} \mathrm{d} s \\
& \left.+\left[\frac{\left|\phi\left(x_{r+1}, t_{k}\right)\right|^{2}-2\left|\phi\left(x_{r}, t_{k}\right)\right|^{2}+\left|\phi\left(x_{r-1}, t_{k}\right)\right|^{2}}{h^{2}}\right] \int_{0}^{h} \frac{s^{2}}{2|(r-j) h+s|^{\alpha}} \mathrm{d} s\right) .
\end{aligned}
$$

with the local truncation error $O\left(h^{3-\alpha}\right)$. Moreover, The integrals in (A.3), (A.4) and (A.5) can be calculated analytically, we omit here for brevity. 


\section{Acknowledgements}

This work was supported by National Natural Science Foundation of China (No. 11171032) and Beijing Municipal Education Commission (Nos. KM201110772017, 71D09111003).

\section{Author details}

${ }^{1}$ School of Applied Science, Beijing Information Science and Technology University, Beijing 100192, P. R. China ${ }^{2}$ National Laboratory for Scientific Computing, Ministry of Science and Technology, Avenida Getulio Vargas 333, 25651075 Petropólis, RJ, Brazil

\section{Authors' contributions}

DY $\mathrm{H}$ established the scheme, performed the numerical examples in section 5.1, 5.2 and drafted the manuscript. XG L designed the study and carried out the the numerical simulations in section 5.3. J Z participated in explaining the physical background, established the model and helped to inspect the manuscript. All authors read and approved the final manuscript.

\section{Competing interests}

The authors declare that they have no competing interests.

Received: 22 April 2012 Accepted: 21 June 2012 Published: 21 June 2012

\section{References}

1. Pitaevskii, L, Stringari, S: Bose-Einstein Condensation. Oxford University, New York (2003)

2. Griesmaier, A, Werner, J, Hensler, S, Stuhler, J, Pfau, T: Bose-Einstein condensation of chromium. Phys Rev Lett 94 (2005). article 160401

3. Stuhler, J, Griesmaier, A, Koch, T, Fattori, M, Pfau, T, Giovanazzi, S, Pedri, P, Santos, L: Observation of dipole-dipole interaction in a degenerate quantum gas. Phys Rev Lett. 95, article 150406 (2005)

4. Baranov, MA, Mar'enko, MS, Rychkov, VS, Shlyapnikov, GV: Superfluid pairing in a polarized dipolar Fermi gas. Phys Rev A 66 (2002). article 013606

5. Góral, K, Santos, L, Lewenstein, M: Quantum phases of dipolar bosons in optical lattices. Phys Rev Lett 88 (2002). article 170406

6. Kawaguchi, Y, Saito, H, Ueda, M: Einstein-de Haas effect in dipolar Bose-Einstein condensates. Phys Rev Lett 96 (2006). article 080405

7. Lahaye, T, Menotti, C, Santos, L, Lewenstein, M, Pfau, T: The physics of dipolar bosonic quantum gases. Rep Prog Phys 72 (2009). article 126401

8. Santos, L, Shlyapnikov, GV, Zoller, P, Lewenstein, M: Bose-Einstein condensation in trapped dipolar gases. Phys Rev Lett. 85, 1791-1794 (2000). doi:10.1103/PhysRevLett.85.1791

9. Yi, S, You, L: Trapped atomic condensates with anisotropic interactions. Phys Rev A 61 (2000). article 041604(R)

10. Yi, S, You, L: Trapped condensates of atoms with dipole interactions. Phys Rev A 63 (2001). article 053607

11. Yi, S, You, L: Calibrating dipolar interaction in an atomic condensate. Phys Rev Lett 92 (2004). article 193201

12. Carles, R, Markowich, PA, Sparber, C: On the Gross-Pitaevskii equation for trapped dipolar quantum gases. Nonlinearity. 21, 2569-2590 (2008). doi:10.1088/0951-7715/21/11/006

13. Bao, W, Cai, Y, Wang, H: Efficient numerical methods for computing ground states and dynamics of dipolar BoseEinstein condensates. J Comput Phys. 229, 7874-7892 (2010). doi:10.1016/j.jcp.2010.07.001

14. Chen, J, Guo, B: Strong instability of standing waves for a nonlocal Schrödinger equation. Physica D. 227, 142-148 (2007). doi:10.1016/j.physd.2007.01.004

15. Lahaye, T, Metz, J, Fröhlich, B, Koch, T, Meister, M, Griesmaier, A, Pfau, T, Saito, H, Kawaquchi, Y, Ueda, M: d-Wave collapse and explosion of a dipolar Bose-Einstein condensate. Phys Rev Lett 101 (2008). article 080401

16. Góral, K, Rzażewski, K, Pfau, T: Bose-Einstein condensation with magnetic dipole-dipole forces. Phys Rev A. 61, article 051601(R) (2000)

17. Ronen, S, Bortolotti, D, Bohn, J: Bogoliubov modes of a dipolar condensate in acylindrical trap. Phys Rev A 74 (2006). article 013623

18. Chiofalo, ML, Succi, S, Tosi, MP: Ground state of trapped interacting Bose-Einstein condensates by an explicit imaginarytime algorithm. Phys Rev E. 62, 7438-7444 (2000). doi:10.1103/PhysRevE.62.7438

19. Bao, W, Du, Q: Computing the ground state solution of Bose-Einstein condensates by a normalized gradinet flow. SIAM J Sci Comput. 25, 1674-1697 (2004). doi:10.1137/S1064827503422956

20. Strang, G: On the construction and comparison of difference schemes. SIAM J Numer Anal. 5, 505-517 (1986)

21. Li, XG, Chan, CK, Hou, Y: A numerical method with particle conservation for the Maxwell-Dirac system. Appl Math Comput. 216, 1096-1108 (2010). doi:10.1016/j.amc.2010.02.002

22. Bao, W, Li, XG: An efficient and stable numerical method for the Maxwell-Dirac system. J Comput Phys. 199, 663-687 (2004). doi:10.1016/j.jcp.2004.03.003

doi:10.1186/1687-1847-2012-85

Cite this article as: Hua et al:: A mass conserved splitting method for the nonlinear Schrödinger equation.

Advances in Difference Equations 2012 2012:85. 